\title{
Is age an independent determinant of mortality in cardiac surgery as suggested by the EuroSCORE?
}

\author{
Amir Mortasawi*1, Bert Arnrich ${ }^{2}$, Ulrich Rosendahl1, Inez Frerichs ${ }^{3}$, \\ Alexander Albert ${ }^{1}$, Jörg Walter ${ }^{2}$ and Jürgen Ennker ${ }^{1}$
}

Address: ${ }^{1}$ Clinic of Thoracic and Cardiovascular Surgery, Heart Institute Lahr/Baden, Lahr, Germany, ${ }^{2}$ Department of Neuroinformatics, University of Bielefeld, Bielefeld, Germany and ${ }^{3}$ Department of Anaesthesiological Research, University of Göttingen, Göttingen, Germany

E-mail: Amir Mortasawi* - a.mortasawi@heart-lahr.com; Bert Arnrich - mail@barnrich.de; Ulrich Rosendahl - ulrich.rosendahl@heart-lahr.com; Inez Frerichs - isipink@gwdg.de; Alexander Albert - alexander.albert@heart-lahr.com; Jörg Walter - walter@techfak.uni-bielefeld.de;

Jürgen Ennker - juergen.ennker@heart-lahr.com

*Corresponding author

Published: 7 October 2002

BMC Surgery 2002, 2:8

This article is available from: http://www.biomedcentral.com/I47/-2482/2/8

(C) 2002 Mortasawi et al; licensee BioMed Central Ltd. This article is published in Open Access: verbatim copying and redistribution of this article are permitted in all media for any purpose, provided this notice is preserved along with the article's original URL.

Keywords: Age, cardiac surgery, EuroSCORE, mortality, morbidity

\begin{abstract}
Background: The proportion of older patients in cardiac surgery is continuously increasing. $37 \%$ of patients undergoing heart surgery in Germany in the year 2000 were 70 years of age and older. We have studied the role of age as a determinant of mortality in cardiac surgery in our institutional patient population.

Methods: We have calculated the EuroSCORE and the corresponding age-adjusted EuroSCORE in 8769 patients who underwent heart surgery between January 1996 and January 2002 and collected the information on the occurrence of postoperative complications and 30-days mortality.

Results: The multimorbidity increased with ascending age. Both the EuroSCORE and the ageadjusted EuroSCORE values increased significantly with age in the whole group of patients as well as in the group of patients who were alive 30 days after heart surgery. The incidence of postoperative complications and 30-days mortality increased significantly with age. In patients who died within 30 days after surgery, the EuroSCORE increased significantly with age, whereas the ageadjusted EuroSCORE did not. The occurrence of diabetes mellitus, arterial hypertension and atrial fibrillation, i.e., the risk factors not considered by the EuroSCORE, exhibited a significant age dependence in our patients. The univariate analysis identified the significant dependence of 30-days mortality on diabetes and atrial fibrillation. The stepwise logistic regression analysis showed the dependence of mortality on diabetes.
\end{abstract}

Conclusions: On the background of the well-known age-dependent structural and functional changes of different body organs, our data show that age is a significant risk indicator in cardiac surgery, strongly correlating with morbidity and mortality. Consequently, special preventive and therapeutic measures are required in clinical environment in the case of elderly patients undergoing cardiac surgery. 


\section{Backgraound}

The proportion of older patients in cardiac surgery is increasing as a consequence of demographic changes and new developments in medical technology. In the year 1989,3673 patients in the age of 70 years and over underwent a cardiac surgical procedure in Germany, whereas in the year 2000 the number of operated patients in this age group increased to 35884 . Thus, the proportion of patients aged 70 years and older increased from 11,2\% to $36,7 \%[1,2]$.

The EuroSCORE is one of the established risk evaluation scores in heart surgery in Europe [3-5]. In Germany, the
EuroSCORE is presently being employed as a nation-wide quality control tool for the assessment of the 30-days mortality risk in cardiac surgery. It is based on a logistic regression model and includes all adult cardiac surgery procedures within a defined 30-days time interval. Besides other parameters, the age above 60 years is regarded as an independent determinant of mortality in this score system, whereby the risk weight is increased in 5-years intervals (table 1; see also [http://www.euroscore.org/ calc.html]). We have checked the effect of age on the mortality rate and the occurrence of postoperative complications in our institutional patients.

Table I: EuroScore (4)

Variable Definition and Scoring

\section{Patient-related factors}

Age (years)
Gender
Chronic pulmonary disease
Extracardiac arteriopathy
Neurological dysfunction
Previous cardiac surgery
Serum creatinine
Active endocarditis
Critical preoperative state

\section{Cardiac-related factors}

Unstable angina

Left ventricular dysfunction

Recent myocardial infarction Pulmonary hypertension

Operation-related factors Emergency

Other than isolated CABG Surgery on thoracic aorta Postinfarction septal rupture Score

$0-2$

$3-5$

$\geq 6$
Per 5 years or part thereof over 60 years

Female

Long term use of bronchodilators or steroids for lung disease Any one or more of the following: claudication, carotid occlusion or $>50 \%$ stenosis, previous or planned intervention on the abdominal aorta, limb arteries or carotids Disease severely affecting ambulation or day-to-day functioning $\quad 2$

Surgery requiring opening of the pericardium

Serum creatinine $>200 \mu \mathrm{mol} / \mathrm{l}$ preoperatively

Patient still on antibiotic treatment for endocarditis at time of surgery

Any one or more of the following: ventricular tachycardia or fibrillation or aborted sudden death, preoperative cardiac massage, preoperative ventilation before anaesthetic room, preoperative inotropes or intra-aortic balloon pump, preoperative acute renal failure (anuria or oliguria $<10 \mathrm{ml} / \mathrm{h}$ )

Rest angina requiring intravenous nitrates until arrival in anaesthetic room

Moderate or left ventricular ejection fraction $30-50 \%$ Poor or left ventricular ejection fraction $<30 \%$

Myocardial infarction within 90 days

Systolic pulmonary artery pressure $>60 \mathrm{mmHg}$

Carried out on referral before the beginning of the next working day

Major cardiac procedure other than or in addition to CABG

For disorders of ascending, arch or descending aorta

Risk

Low

Medium

High
I

I 2 3 2 3

3 2 13 2 2 2 2 3 4 Expected mortality ( $95 \%$ confidence limits)

I,27-I,29\%

$2,90-2,94 \%$

$10,93-\mid 1,54 \%$ 
Table 2: Preoperative data

\begin{tabular}{|c|c|c|c|c|c|c|c|c|}
\hline Age group & $<60$ & $61-65$ & $66-70$ & $71-75$ & $76-80$ & $81-85$ & $86-90$ & $P$ value \\
\hline$n$ & 2180 & 1539 & 1798 & $|73|$ & 1169 & 293 & 58 & --- \\
\hline Female & $15,6 \%$ & $22,2 \%$ & $27,0 \%$ & $38,1 \%$ & $45,9 \%$ & $59,7 \%$ & $56,9 \%$ & $<0,005$ \\
\hline COPD & $2,8 \%$ & $3,8 \%$ & $4,6 \%$ & $5.0 \%$ & $5,3 \%$ & $7,2 \%$ & $8,6 \%$ & $<0,005$ \\
\hline Extracardiac arteriopathy & $10,6 \%$ & $15,7 \%$ & $17,7 \%$ & $19,2 \%$ & $18,8 \%$ & $15,7 \%$ & $20,7 \%$ & $<0,005$ \\
\hline Neurological dysfunction & $2,6 \%$ & $3,0 \%$ & $4,7 \%$ & $4,3 \%$ & $4,0 \%$ & $5,5 \%$ & $1,7 \%$ & 0,006 \\
\hline Previous cardiac surgery & $4,8 \%$ & $4,8 \%$ & $5,6 \%$ & $4,6 \%$ & $4,4 \%$ & $3,1 \%$ & $5,2 \%$ & 0,658 \\
\hline Serum creatinine $>200 \mu \mathrm{mol} / \mathrm{l}$ & $1,6 \%$ & $1,6 \%$ & $\mathrm{I}, 2 \%$ & $2,5 \%$ & $1,8 \%$ & $0,7 \%$ & $5,2 \%$ & 0,038 \\
\hline Active endocarditis & $\mathrm{I}, 2 \%$ & $0,5 \%$ & $0,3 \%$ & $0,7 \%$ & $0,3 \%$ & $0,7 \%$ & $1,7 \%$ & 0,014 \\
\hline Critical preoperative state & $0,8 \%$ & $0,5 \%$ & $0,6 \%$ & $0,7 \%$ & $0,4 \%$ & $1,0 \%$ & $\mathrm{I}, 7 \%$ & 0,777 \\
\hline Unstable angina & $9,4 \%$ & $10,7 \%$ & $11,2 \%$ & $9,4 \%$ & $11,8 \%$ & $14,0 \%$ & $27,0 \%$ & $<0,005$ \\
\hline LV ejection fraction $30-50 \%$ & $28,4 \%$ & $28,0 \%$ & $33,0 \%$ & $34,4 \%$ & $33,5 \%$ & $37,2 \%$ & $53,4 \%$ & $<0,005$ \\
\hline LV ejection fraction $<30 \%$ & $4,3 \%$ & $4,8 \%$ & $5,6 \%$ & $4,7 \%$ & $5,5 \%$ & $4,4 \%$ & $6,9 \%$ & 0,635 \\
\hline Recent myocardial infarct & $\mathrm{II}, \mathrm{l} \%$ & $9,4 \%$ & $9,9 \%$ & $9,5 \%$ & $7,4 \%$ & $9,2 \%$ & $3,4 \%$ & 0,034 \\
\hline Pulmonary hypertension & $5,1 \%$ & $4,5 \%$ & $4,5 \%$ & $6,4 \%$ & $6.0 \%$ & $8,5 \%$ & $6,9 \%$ & 0,032 \\
\hline Emergency & $1,4 \%$ & $1,3 \%$ & $\mathrm{I}, \mathrm{l} \%$ & I,7\% & $\mathrm{I}, 5 \%$ & $\mathrm{I}, 7 \%$ & $5,2 \%$ & 0,309 \\
\hline Other than isolated $C A B G$ & $15,0 \%$ & $1 \mathrm{l}, 4 \%$ & $10,4 \%$ & $12,4 \%$ & $15,0 \%$ & $23,5 \%$ & $24,1 \%$ & $<0,005$ \\
\hline Surgery on thoracic aorta & $2,6 \%$ & $1,2 \%$ & $1,0 \%$ & $1,2 \%$ & $0,5 \%$ & $0,7 \%$ & $0,0 \%$ & $<0,005$ \\
\hline Postinfarction septal rupture & $0,0 \%$ & $0,1 \%$ & $0,0 \%$ & $0,0 \%$ & $0,0 \%$ & $0,0 \%$ & $0,0 \%$ & 0,157 \\
\hline Arterial hypertension & $62,2 \%$ & $70,1 \%$ & $74,0 \%$ & $74,6 \%$ & $71,7 \%$ & $67,2 \%$ & $100 \%$ & $<0,005$ \\
\hline Diabetes mellitus & $20,1 \%$ & $27,6 \%$ & $28,8 \%$ & $29,6 \%$ & $29,9 \%$ & $27,6 \%$ & $17,2 \%$ & $<0,005$ \\
\hline Atrial fibrillation & $2,9 \%$ & $4,8 \%$ & $6,1 \%$ & $9,5 \%$ & $9,8 \%$ & $14,7 \%$ & $13,8 \%$ & $<0,005$ \\
\hline
\end{tabular}

\section{Methods}

Several patient files, for instance, the databases of the departments of anaesthesiology, clinical laboratory and institutional management, the database of the nation-wide quality control in cardiac surgery, internal follow-up information files and the databases of specific groups of patients, e.g. the patients requiring haemodialysis, are assembled in parallel in our institution. In cooperation with the Department of Neuroinformatics at the University in Bielefeld, we have created a new specialized database, bringing together the information from the already existing database systems. Using this specialized database, we have retrospectively studied a total of 8769 patients who underwent a cardiac surgery in the time interval between January 1996 and January 2002.

According to the established EuroSCORE evaluation procedure, our patients were divided into seven age groups. The EuroSCORE and the age-adjusted EuroSCORE values were calculated for each patient, in the latter case by subtracting the scoring points for the corresponding age group. Thereafter, the occurrence of postoperative complications and the 30-days mortality were determined. Finally, statistical analyses (Pearson' Chi square test, ANOVA) were performed with the aim to establish the role of age in cardiac surgery. Statistical significance was defined as $p$ $<0.05$. In addition to the EuroSCORE parameters, we have also determined the occurrence of arterial hyperten- sion, diabetes mellitus, as well as atrial fibrillation in all age groups studied. The dependence of the 30-days mortality on these three variables was checked by univariate as well as stepwise logistic regression analyses.

\section{Results}

The calculated EuroSCORE parameters in all age groups studied, as well as the occurrence of the three additionally determined accompanying diseases, are shown in table 2. Highly significant age-dependent differences in the distribution of the following variables were found: female gender, chronic obstructive pulmonary disease (COPD), noncardiac atherosclerosis, neurological dysfunction, instable angina, left ventricular ejection fraction $30-50 \%$, non-isolated coronary artery bypass grafting (CABG), thoracic aortic surgery, arterial hypertension, diabetes and atrial fibrillation. Table 3 shows the EuroSCORE and the age-adjusted EuroSCORE values in the following three groups of patients: 1 . all patients, 2 . patients who were alive 30 days after surgery and 3 . patients who died within 30 days after surgery. Both the EuroSCORE and the age-adjusted EuroSCORE values increased significantly with age in the group of all patients studied and in the group of 30-days survivors. In the group of patients who died within 30 days after cardiac surgery, the EuroSCORE exhibited a significant age-dependent increase, however, the age-adjusted EuroSCORE value did not change significantly with age. 
Table 3: EuroScore and age-stripped EuroScore

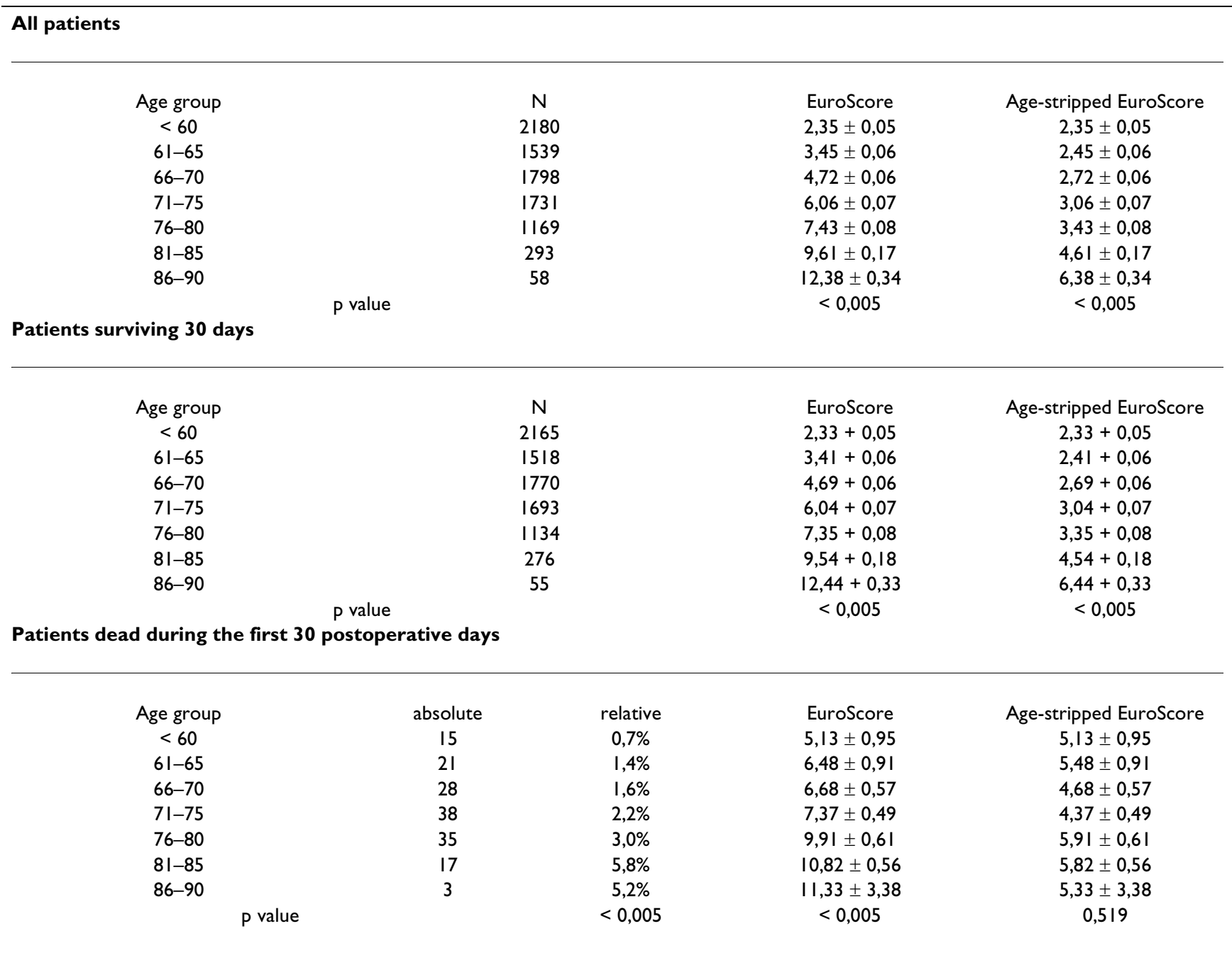

Tables 4 and 5 summarize the surgical procedures performed in the seven age groups studied and show the corresponding 30-days mortalities. The relative number of coronary bypass surgeries, with a mortality rate of $1,1 \%$, decreased with age in our patient population, whereas the number of aortic valve replacement procedures, either with or without accompanying coronary grafting, increased. The mortality for aortic valve replacement was $2,7 \%$ and $4,1 \%$ in the case of isolated and combined surgical procedures, respectively. Table 6 shows the incidence of postoperative complications. The 30-days mortality and the occurrence of most postoperative complications rose significantly with increasing age. The univariate analysis revealed a significant dependence of 30-days mortality on diabetes $(\mathrm{p}=0,016)$ and atrial fibrillation $(\mathrm{p}<$ $0,005)$. The stepwise logistic regression analysis showed a significant dependence of mortality on diabetes $(\mathrm{p}=$ 0,050).

\section{Discussion}

The demographic changes result in a continuously increasing number of elderly patients being treated in cardiac surgery departments. In Germany, there exists no nation-wide database on the prevalence and incidence of those accompanying diseases studied in our patient group for the whole population, therefore, it is not possible to compare our patients with an age-matched group of subjects to determine any possible biological selection. If we consider the age-adjusted EuroSCORE as a measure of multimorbidity, then our data show an increase in multimorbidity in patients undergoing cardiac surgery with age. A detailed analysis of the individual EuroSCORE variables and the additional evaluation of other parameters confirm that the number of accompanying diseases in patients undergoing cardiac surgery increases with age. When compared with other age groups, the relatively small number of patients aged 80 years and over in our 
Table 4: Operative procedures

\begin{tabular}{|c|c|c|c|c|c|c|c|c|c|c|c|c|c|c|}
\hline \multirow[t]{2}{*}{ Age group } & \multicolumn{2}{|c|}{$<60$} & \multicolumn{2}{|c|}{$61-65$} & \multicolumn{2}{|c|}{$65-70$} & \multicolumn{2}{|c|}{$7 I-75$} & \multicolumn{2}{|c|}{$76-80$} & \multicolumn{2}{|c|}{$81-85$} & \multicolumn{2}{|c|}{$86-90$} \\
\hline & $\mathrm{n}$ & $\%$ & $n$ & $\%$ & $\mathrm{n}$ & $\%$ & $\mathrm{n}$ & $\%$ & $\mathrm{n}$ & $\%$ & $n$ & $\%$ & $\mathrm{n}$ & $\%$ \\
\hline All procedures & 2180 & 100 & 1539 & 100 & 1798 & 100 & $173 \mid$ & 100 & 1169 & 100 & 293 & 100 & 58 & 100 \\
\hline CABG & 1554 & 71,28 & 1126 & 73,16 & 1295 & 72,02 & 1185 & 68,46 & 739 & 63,22 & 132 & 45,05 & 26 & 44,83 \\
\hline AVR & 145 & 6,65 & 100 & 6,49 & 117 & 6,51 & 124 & 7,16 & 127 & 10,86 & 52 & 17,74 & 10 & 17,24 \\
\hline$A V R+C A B G$ & 62 & 2,84 & 56 & 3,64 & 102 & 5,67 & 118 & 6,82 & 128 & 10,95 & 60 & 20,48 & 13 & 22,41 \\
\hline MVR & 75 & 3,44 & 36 & 2,34 & 32 & 1,78 & 37 & 2,14 & 20 & $1,7 \mid$ & 9 & 3,07 & 2 & 3,45 \\
\hline$A V R+M V R$ & 10 & 0,46 & 5 & 0,32 & 5 & 0,28 & 15 & 0,87 & 7 & 0,59 & 1 & 0,34 & 0 & 0 \\
\hline$M V R+C A B G$ & 22 & $|, 0|$ & 15 & 0,97 & 29 & 1,61 & 28 & 1,62 & 27 & 2,31 & 6 & 2,05 & 0 & 0 \\
\hline OPCAB & 98 & 4,49 & 74 & 4,81 & 69 & 3,84 & 73 & 4,22 & 30 & 2,57 & 18 & 6,14 & 4 & 6,89 \\
\hline Other & 214 & 9,82 & 127 & 8,25 & 149 & 8,29 & $15 \mid$ & 8,72 & 91 & 7,78 & 15 & 5,12 & 3 & 5,17 \\
\hline
\end{tabular}

CABG: coronary artery bypass grafting, AVR: aortic valve replacement, MVR: mitral valve repair or replacement, OPCAB: off-pump coronary artery bypass grafting, Other: redo procedures, surgery on thoracic aorta, repair of atrial septal defect, repair of postinfarction septal defect

Table 5: 30-days mortality

\begin{tabular}{lccccccccc}
\hline Age group & $<60$ & $61-65$ & $66-70$ & $71-75$ & $76-80$ & $81-85$ & $86-90$ & $\begin{array}{c}\text { P value } \\
\text { groups }\end{array}$ \\
\hline All procedures & $0,7 \%$ & $1,4 \%$ & $1,6 \%$ & $2,2 \%$ & $3,0 \%$ & $5,8 \%$ & $5,2 \%$ & $<0,005$ \\
CABG & $0,5 \%$ & $1,0 \%$ & $0,8 \%$ & $1,4 \%$ & $2,4 \%$ & $1,5 \%$ & $3,8 \%$ & $<0,005$ & $1,8 \%$ \\
AVR & $0,7 \%$ & $3,0 \%$ & $3,4 \%$ & $1,6 \%$ & $3,1 \%$ & $7,7 \%$ & 0 & 0,206 & $2,7 \%$ \\
AVR+CABG & 0 & $1,8 \%$ & $3,9 \%$ & $4,2 \%$ & $2,3 \%$ & $11,7 \%$ & $15,4 \%$ & 0,014 & $4,1 \%$ \\
MVR & $1,3 \%$ & $2,8 \%$ & $3,1 \%$ & $5,4 \%$ & $15,0 \%$ & $11,1 \%$ & 0 & 0,188 & $4,3 \%$ \\
AVR+MVR & $10,0 \%$ & 0 & 0 & $6,7 \%$ & $14,3 \%$ & $100 \%$ & -- & 0,049 & $9,3 \%$ \\
MVR+CABG & $9,1 \%$ & 0 & 0 & $3,6 \%$ & $3,7 \%$ & 0 & --- & 0,519 & $3,1 \%$ \\
OPCAB & 0 & 0 & $1,5 \%$ & $2,7 \%$ & $3,3 \%$ & $5,6 \%$ & 0 & 0,352 & $1,4 \%$ \\
Other & $1,4 \%$ & $3,9 \%$ & $4,7 \%$ & $6,0 \%$ & $4,4 \%$ & $6,7 \%$ & 0 & 0,416 & $3,9 \%$ \\
& & & & & & & & & \\
\hline
\end{tabular}

CABG: coronary artery bypass grafting, AVR: aortic valve replacement, MVR: mitral valve repair or replacement, OPCAB: off-pump coronary artery bypass grafting, Other: redo procedures, surgery on thoracic aorta, repair of atrial septal defect, repair of postinfarction septal defect

patient population allow only limited conclusions to be drawn from the data obtained in this specific group of patients. Alexander et al. [6] found the following significant differences between a group of 60161 patients aged below 80 years and undergoing an isolated CABG in comparison with a group of 4306 patients 80 years of age and older: female gender $28,2 \%$ vs. $44,1 \%$, COPD $16,0 \%$ vs $14,1 \%$, diabetes mellitus $29,5 \%$ vs. $23,0 \%$, renal insufficiency $4,8 \%$ vs. $8,0 \%$, chronic cardiac failure $11,7 \%$ vs. $19,4 \%$, cerebrovascular disease $10,4 \%$ vs. $18,7 \%$, peripheral vascular disease $13,7 \%$ vs. $16,4 \%$, coronary triple vessel disease $64,3 \%$ vs. $70,4 \%$. Also other studies indicate that age increases the multimorbidity in patients undergoing heart surgery $[7,8]$. If we take this increasing multimorbidity into account, then the rise in 30-days mortality and occur- rence of most postoperative complications with age, observed in our patient population, is not an unexpected result. Moreover, it is in harmony with the results of other research groups [6-9]. Also the reduction of the number of isolated coronary surgeries with increasing age, observed in our group of patients, corresponds with the findings of other authors $[6,8]$. This surgical procedure is associated with a lower overall mortality when compared with the valve replacement and repair, as well as combined surgical procedures, nevertheless, it contributes to the increasing mortality with age. In the year 2000, the mortality for isolated CABG in Germany was 2,9\%, 3,3\% for isolated aortic valve replacement and 6,0\% for simultaneous coronary bypass surgery with accompanying aortic valve replacement [2]. This issue is considered in the 
EuroSCORE evaluation in the risk factor called "other than isolated CABG".

In the patients, who died within 30 days after surgery, the EuroSCORE rose significantly with age, however, the score value did not show such significant changes after adjustment for age. Therefore, the question arises what is the reason for the increased mortality observed in the elderly and, further, what other factors are concealed behind the common variable 'age'. The following four points have to be discussed with respect to this issue:

1. Several diseases, e.g., COPD, peripheral atherosclerosis or renal insufficiency, are considered in the EuroSCORE analysis, however, the stage of the respective diseases and the intensity of the pathological processes, both of which are influenced by the time factor, are not taken into account. Therefore, the different intensity and duration of the mentioned diseases may be hidden in the EuroSCORE parameter 'age'.

2. The EuroSCORE risk evaluation system has been derived from a large database of cardiac surgical patients. Some diseases were not identified as being relevant with respect to mortality in this large patient population by the multivariate analysis and, therefore, were not considered during the generation of the EuroSCORE list of risk factors. We have studied the dependence of mortality on arterial hypertension, diabetes and atrial fibrillation, i.e., the three factors not included in the EuroSCORE system. All three factors exhibited significant differences in their respective age-related distributions. The univariate analysis determined a significant dependence of the 30-days mortality on diabetes and atrial fibrillation. The multivariate analysis revealed the dependence of the 30-days mortality on diabetes. The effect of such factors, that are not separately considered in the EuroSCORE analysis, may also partly contribute to the parameter 'age'.

3. The following factors are taken into account by the EuroSCORE system to characterize the cardiac status: instable angina, left ventricular ejection fraction $30-50 \%$ or $<30 \%$, myocardial infarction within preceding 90 days before surgery, systolic pulmonary artery pressure $>60 \mathrm{~mm}$ Hg. These patient data guarantee objective data acquisition and good comparability among hospitals with differing personnel and technology backgrounds and adequately describe certain aspects of the cardiac status. It would be inappropriate to expect the EuroSCORE system to consider in detail all characteristic features of the cardiovascular system in its complexity. However, one factor, shown to possess a high degree of diagnostic and therapeutic relevance in the elderly, the diastolic cardiac function [10], is not taken into account in the EuroSCORE cardiac operative risk evaluation. Senni et al. [11] have found out that $43 \%$ of patients with the primary diagnosis of cardiac failure exhibited normal systolic heart function. Other studies have shown that diastolic heart failure is found in about $50 \%$ of elderly patients suffering from congestive heart failure $[12,13]$. This means, that also this aspect may be hidden in the EuroSCORE parameter 'age'.

Table 6: Postoperative complications

\begin{tabular}{|c|c|c|c|c|c|c|c|c|}
\hline Age group & $<60$ & $61-65$ & $66-70$ & $71-75$ & $76-80$ & $81-85$ & $86-90$ & $\mathrm{P}$ value \\
\hline $\mathrm{n}$ & 2180 & 1539 & 1798 & |73| & 1169 & 293 & 58 & --- \\
\hline Pneumonia & $7,8 \%$ & $8,6 \%$ & $9,0 \%$ & $11,6 \%$ & $11,7 \%$ & $18,1 \%$ & $6,9 \%$ & $<0,005$ \\
\hline Arrhythmia & $31,6 \%$ & $39,1 \%$ & $48,2 \%$ & $54,7 \%$ & $60,5 \%$ & $67,2 \%$ & $70,7 \%$ & $<0,005$ \\
\hline Myocardial infarction & $1,2 \%$ & $1,2 \%$ & I,7\% & $1,8 \%$ & $1,7 \%$ & $2,7 \%$ & $1,7 \%$ & 0,493 \\
\hline Unstable sternum & $0,3 \%$ & $0,6 \%$ & $\mathrm{I}, \mathrm{l} \%$ & $0,9 \%$ & $1,1 \%$ & $1,4 \%$ & $5,2 \%$ & 0,001 \\
\hline Significant pleural effusion & $9,0 \%$ & I I,0\% & I I,3\% & $13,5 \%$ & $15,2 \%$ & $16,0 \%$ & $15,5 \%$ & $<0,005$ \\
\hline Significant pericardial effusion & $0,6 \%$ & $0,8 \%$ & $0,8 \%$ & $1,2 \%$ & $1,5 \%$ & $2,7 \%$ & $1,7 \%$ & 0,016 \\
\hline Cerebral vascular accident & $\mathrm{I}, 7 \%$ & $1,7 \%$ & $2,1 \%$ & $3,6 \%$ & $3,8 \%$ & $6,1 \%$ & $8,6 \%$ & $<0,005$ \\
\hline Confusion & $2,0 \%$ & $4,2 \%$ & $5,8 \%$ & $9,3 \%$ & $13,1 \%$ & $21,2 \%$ & $24,1 \%$ & $<0,005$ \\
\hline Rethoracotomy & $2,6 \%$ & $2,8 \%$ & $3,2 \%$ & $3,4 \%$ & $3,3 \%$ & $5,5 \%$ & $5,2 \%$ & 0,238 \\
\hline Reintubation & $2,5 \%$ & $3,0 \%$ & $4,5 \%$ & $4,4 \%$ & $7,0 \%$ & $7,8 \%$ & $10,3 \%$ & $<0,005$ \\
\hline Resuscitation & $\mathrm{I}, 8 \%$ & $1,8 \%$ & $2,6 \%$ & $2,6 \%$ & $3,0 \%$ & $7,5 \%$ & I,7\% & $<0,005$ \\
\hline IABP & $0,7 \%$ & $0,6 \%$ & $0,6 \%$ & $0,9 \%$ & I, I\% & $1,4 \%$ & 0 & 0,622 \\
\hline Dialysis & $1,6 \%$ & $2,8 \%$ & $3,0 \%$ & $4,0 \%$ & $6,4 \%$ & $7,8 \%$ & $13,8 \%$ & $<0,005$ \\
\hline Laparotomy & $0,1 \%$ & $0,1 \%$ & $0,3 \%$ & $0,1 \%$ & $0,3 \%$ & $0,3 \%$ & 0 & 0,399 \\
\hline 30-days mortality & $0,7 \%$ & $1,4 \%$ & $1,6 \%$ & $2,2 \%$ & $3,0 \%$ & $5,8 \%$ & $5,2 \%$ & $<0,005$ \\
\hline
\end{tabular}


4. Finally, the aging process is associated with structural and functional changes in various organ systems, which may influence the perioperative outcome. The following age-dependent changes in the cardiovascular system have previously been identified: dilation of large vessels, thickening of vessel walls, affecting first of all the intimal layer, loss of vessel elasticity [14-16], increase in left ventricular afterload [17], thickening of left ventricular wall [14,18], cardiac myocyte cell loss and increase in the amount of myocardial collagen [19], decline in early diastolic left ventricular filling rate [20], increased atrial contribution to ventricular filling and larger atrial diameter [18], reduced adaptability of the cardiac response to different workloads resulting from modified ventricular filling volumes and changes in heart rate [17], increasing deficits in sympathetic modulation [21], changes in myocardial calcium hemostasis, affecting the excitation-contraction coupling and, consequently, the myocardial contractility and tendency to arrhythmias $[22,23]$. The consequence of all the above-mentioned changes is the reduced cardiac adaptability to workload observed in the elderly $[24,25]$.

The age-dependent changes in other than cardiovascular body organ systems require specific preventive and therapeutic measures. For instance, the well-known age-related changes in lung structure and function (e.g., increase in functional residual capacity and residual volume, weakness of the expiratory muscles, malfunction of the airway epithelium accompanied with increased production of mucus, diminished tendency to cough, increased ventilation-perfusion mismatch) may lead to complications in older patients undergoing cardiac surgery. To prevent pulmonary complications in the elderly, it is necessary to examine the lung function and to initiate chest physiotherapy with breathing exercise already preoperatively. Early extubation, mobilisation, and pharmacologically supported bronchial clearance should follow postoperatively and a dehydration of the patients should be avoided. (A review of other therapeutic measures in elderly cardiac surgical patients is given in [26].)

\section{Conclusions}

It can be summarized that age strongly correlates with a cluster of risk factors and organ dysfunctions, occurring during aging. However, the old age as such is not a disease. It is only a state characterized by a relatively high probability of suffering from various disturbances of the normal body function. From this point of view, age is a risk factor strongly correlating with mortality. Because of increased occurrence of age-related multimorbidity and changes in organ structure and function, special attention has to be paid to adequate therapeutic measures in elderly patients undergoing heart surgery.

\section{Competing interests}

None declared.

\section{Authors' Contributions}

A. Mortasawi designed the study and drafted the manuscript. B. Arnrich and J. Walter participated in the design of the study and performed the statistical analysis. U. Rosendahl, I. Frerichs, A. Albert, and J. Ennker participated in the design of the study. All authors read and approved the final manuscript.

\section{References}

I. Kalmar P, Irrgang E: Cardiac surgery in the Federal Republic of Germany during 1989. A report by the German Society for Thoracic and Cardiovascular Surgery. Thorac Cardiovasc Surg 1990, 38: 1 $98-200$

2. Kalmar P, Irrgang E: Cardiac surgery in Germany during 2000. A report by the German Society for Thoracic and Cardiovascular Surgery. Thorac Cardiovasc Surg 200I, 48:XXXIII-XXXVIII

3. Geissler HJ, Hölzl P, Marohl S, Kuhn-Regnier F, Mehlhorn U, Südkamp $M$, de Vivie ER: Risk stratification in heart surgery: comparison of six score systems. Eur J Cardiothorac Surg 2000, 17:400-406

4. Nashef SAM, Roques F, Michel P, Gauducheau E, Lemeshow S, Salamon $R$, the EuroSCORE study group: European system for cardiac operative risk evaluation (EuroSCORE). Eur J Cardiothorac Surg 1999, 16:9-13

5. Roques F, Nashef SAM, Michel P, Gauducheau E, de Vinvetiis C, Baudet E, Cortina J, David M, Faichney A, Gabrielle F, Gams E, Harjula A, Totnes MT, Pinna Pintor P, Salamon R, Thulin L: Risk factors and outcome in European cardiac surgery: analysis of the EuroSCORE multinational database of 19030 patients. Eur J Cardiothoracic Surg 1999, 15:816-823

6. Alexander KP, Anstrom KJ, Muhlbaier LH, Grosswald RD, Smith PK, Jones $\mathrm{RH}$, Peterson ED: Outcomes of cardiac surgery in patients age $\geq \mathbf{8 0}$ years: results from the National Cardiovascular Network. J Am Coll Cardiol 2000, 35:73I-738

7. Craver JM, Puskas JD, Weintraub WW, Shen Y, Guyton RA, Gott JP, Jones EL: 601 octogenarians undergoing cardiac surgery: outcome and comparison with younger age groups. Ann Thorac Surg 1999, 67:1104-1110

8. Fruitman DS, MacDougall CE, Ross DB: Cardiac surgery in octogenarians: can elderly patients benefit? Quality of life after cardiac surgery. Ann Thorac Surg 1999, 68:2129-2135

9. Avery GJ, Ley SJ, Hill JD, Hershon JJ, Dick SE: Cardiac surgery in the octogenarian: evaluation of risk, cost, and outcome. Ann Thorac Surg 2001, 71:591-596

10. Kitzman DW: Why is diastolic heart failure in older patients the cardiologist's enigma? Dialogues in Cardiovascular Medicine 2001, 6:95-103

II. Senni M, Tribouilloy CM, Rodeheffer RJ, Jacobsen SJ, Evans JM, Bailey $K R$, Redfield MM: Congestive heart failure in the community. A study of all incident cases in Olmsted County, Minnesota, in 1991. Circulation 1998, 98:2282-2289

12. Cowie MR, Wood DA, Coats AJ, Thompson SG, Poole-Wilson PA, Suresh V, Sutton GC: Incidence and aetiology of heart failure; a population-based study. Eur Heart J 1999, 20:421-428

13. Kitzman DW, Gardin JM, Gottdiener JS, Arnold A, Boineau R, Aurigemma G, Marino EK, Lyles M, Cushman M, Enright PL: Cardiovascular Health Study Research Group. Importance of heart failure with preserved systolic function in patients $\geq 60$ years of age. Am / Cardiol 200I, 87:4I 3-419

14. Gerstenblith G, Frederiksen J, Yin FC, Fortuin NJ, Lakatta EG, Weisfeldt ML: Echocardiographic assessment of a normal adult aging population. Circulation 1977, 56:273-278

15. Levy Bl: Artery changes with aging: degeneration or adaptation? Dialogues in Cardiovascular Medicine 200I, 6: I04-III

16. Nagai Y, Metter EJ, Earley CJ, Kemper MK, Becker LC, Lakatta EG, Fleg JL: Increased carotid artery intimal-medial thickness in asymptomatic older subjects with exercise-induced myocardial ischemia. Circulation 1998, 98:1504-1509

17. Fleg JL, O'Connor F, Gerstenblith G, Becker LC, Clulow J, Schulman SP, Lakatta EG: Impact of age on the cardiovascular response 
to dynamic upright exercise in healthy men and women. I Appl Physiol 1995, 78:890-900

18. Swinne CJ, Shapiro EP, Lima SD, Fleg JL: Age-associated changes in left ventricular diastolic performance during isometric exercise in normal subjects. Am J Cardiol 1992, 69:823-826

19. Olivetti G, Melissari M, Capasso JM, Anversa P: Cardiomyopathy of the aging human heart. Myocyte loss and reactive cellular hypertrophy. Circ Res 1991, 68:1560-1568

20. Schulman SP, Lakatta EG, Fleg JL, Lakatta L, Becker LC, Gerstenblith G: Age-related decline in left ventricular filling at rest and exercise. Am J Physiol 1992, 263:H $1932-\mathrm{H} 1938$

21. Lakatta EG: Deficient neuroendocrine regulation of the cardiovascular system with advancing age in healthy humans. Circulation 1993, 87:631-636

22. Escande D, Coulombe A, Faivre JF, Deroubaix E, Coraboeuf E: Two types of transient outward currents in adult human atrial cells. Am J Physiol 1987, 252:HI 42-HI48

23. Lakatta EG: Cardiovascular regulatory mechanism in advanced age. Physiol Rev 1993, 73:413-467

24. Isoyama S: Age-related changes before and after imposition of hemodynamic stress in the mammalian heart. Life Sci 1996, 58: $1601-1614$

25. Lakatta EG: Cardiovascular aging without a clinical diagnosis. Dialogues in Cardiovascular Medicine 2001, 6:67-91

26. Vaska PL: Cardiac surgery in special populations, part I: Octogenarians, patients with neuropsychiatric disorders, and blacks. AACN Clinical Issues 1997, 8:50-58

\section{Pre-publication history}

The pre-publication history for this paper can be accessed here:

http://www.biomedcentral.com/1471-2482/2/8/prepub
Publish with BioMed Central and every scientist can read your work free of charge

"BioMedcentral will be the most significant development for disseminating the results of biomedical research in our lifetime."

$$
\text { Paul Nurse, Director-General, Imperial Cancer Research Fund }
$$

Publish with BMC and your research papers will be:

- available free of charge to the entire biomedical community

- peer reviewed and published immediately upon acceptance

- cited in PubMed and archived on PubMed Central

- yours - you keep the copyright
BioMedcentral.com editorial@biomedcentral.com 